\title{
Comunicação
}

\section{Desenvolvimento puberal em meninas tratadas de LLA}

\author{
I.M.M. Monteiro, A.J . Bedone, C.L.B. Pinto, S.R. Brandalise \\ Departamento de Tocoginecologia - Faculdade de Ciências Médicas - Centro de Atencão Integral à Saúde da Mulher (CAISM) - \\ UNICAMP, e Centro I nfantil de I nvestigações Hematológicas “Dr. Domingos A. Boldrini”, Campinas, SP.
}

\begin{abstract}
RESUMO - Ов EтIVo. Com o objetivo de avaliar o desenvolvimento puberal após o tratamento de leucemia linfóide aguda (LLA) na infância, foi realizado um estudo retrospectivo, em meninas tratadas de janeiro de 1980 a janeiro de 1991, no Centro de Investi gações Hematológicas "Dr. Domingos A. Boldrini", em Campinas-SP.

Casuistica e Método. Foram selecionadas 42 meninas, tratadas antes da puberdade com quimioterapia sistêmica e intratecal e radioterapia cranial, utilizando doses de 18 ou 24 Grays (Gy).

Resultados. As idades médias da telarca, pubarca e menarca foram inferiores às do grupocontrole, embora com significância estatística
\end{abstract}

\section{NTRODUÇÃO}

Dos vários tipos de câncer que atingem a infância e adol escência, a leucemia linfói de aguda (LLA) é um dos mais comuns. Os avanços no tratamento da doença, com emprego de drogas quimioterápicas e a associação de terapia em SNC para profilaxia da invasão leucêmica do sistema nervoso central (SNC), aumentaram as taxas de sobrevida em crianças, de $20 \%$ para $65 \%$ nas últimas três décadas, chegando, em alguns Serviços, a $90 \%$ para os pacientes de baixo risco ${ }^{1,2}$. Esse fato tem possi bilitado a observação de efeitos colaterais em longo prazo, decorrentes do tratamento realizado.

Alguns estudos encontraram maior incidência de puberdade precoce, notadamente em meninas. A explicação mais provável é que a irradiação craniana pode, em alguns casos, estimular o eixo hipotálamo-hipófise ${ }^{3}$. Em estudo realizado com 255 crianças tratadas de LLA com quimi oterapia e radi oterapia, cuja idade média por ocasião do tratamento era de 3,9 anos para as meninas e de 4,8 anos para os meninos, observou-se puberdade precoce (mais de dois desvios-padrões da média) em $20 \%$ das meninas ( 24 de 121 ) e em aproximadamente $3 \%$ dos meninos ${ }^{3}$. Em outro estudo, compilando dados da literatura, observou-se que a freqüência de puberdade precoce variou entre $10 \%$ e $20 \%$ em meninas com LLA submetidas a tratamento com radioterapia craniana 4 . apenas para a idade da telarca. Não houve diferenças entre os grupos tratados com 18 ou 24Gy. As meninas tratadas antes dos cinco anos de idade apresentaram idade média da menarca estatisticamente inferior àquelas tratadas após cinco anos e em relação ao grupo-controle.

Conclusão. Os resultados mostraram que o desenvolvimento puberal em meninas tratadas de LLA na infância foi mais precoce que o de meninas saudáveis.

UNITERMOS: Puberdade precoce. Leucemia linfóide aguda - tratamento.

Mesmo quando não se observa puberdade precoce (o aparecimento de al gum sinal de maturidade sexual secundária antes dos oito anos de idade) para meninas ${ }^{5}$, pode-se notar, em alguns estudos, precocidade no desenvolvimento puberal. A idade da menarca em meninas tratadas de LLA foi de 12,2 anos, contra 13,1 anos da população geral na Suécia, sendo esta diferença estatisticamente significativa 6 .

Em outros estudos, tem sido observado atraso puberal em meninas tratadas de LLA com uma incidência maior que na população geral. Essa alteração tem sido notada mais freqüentemente com doses de radioterapia superiores a 35Gy4. Quando utilizadas doses entre 18 e 24Gy, foi mais comum o desenvolvimento de puberdade precoce ${ }^{3}$.

Permanecem controvérsias, no entanto, pois em estudo realizado com 52 crianças holandesas, tratadas de LLA, com dose de 25Gy para irradiação cranial, não se observou puberdade precoce quando o tratamento foi realizado antes dos sete anos de idade; quando o tratamento foi iniciado após os sete anos, a idade da menarca foi maior que a da população geral ${ }^{7}$.

Além da radioterapia, a quimioterapia pode determinar alterações no desenvolvimento puberal, provavelmente por seu efeito sobre as gônadas. A lesão gonadal após a quimioterapia está bem documentada em adultos ${ }^{8}$. Em mulheres mais jovens, os efeitos da quimioterapia trazem menos conseqüências. Observou-se alteração da função ovaria- 
na em seis de 18 pacientes pós-púberes tratadas de leucemia aguda, uma proporção superior à observada em meninas pré-púberes ${ }^{9}$.

Os resultados obtidos em estudos com crianças têm sido conflitantes ${ }^{8}$; alguns têm demonstrado desenvolvimento puberal e função gonadal normais ${ }^{9-11}$ e, outros, vários graus de lesão gonadal ${ }^{12-14}$.

E $m$ relação ao desenvolvimento puberal, parece que são fatores importantes tanto a dose de radioterapia como a idade do tratamento. Com o objetivo de avaliar o desenvolvimento puberal após o tratamento de LLA, foi realizado um estudo em meninas que não apresentaram sinais de recaída da doença após o tratamento.

\section{SUJ EITOS E MÉTODO}

Foi desenvolvido um estudo descritivo e retrospectivo que envolveu prontuários de meninas tratadas de LLA, no período de 1을 de janeiro de 1980 a 31 de janeiro de 1991, com diagnóstico confirmado citol ogicamente através de mi el ograma, atendidas no Centro de Investigações Hematológicas "Dr. Domingos A. Boldrini", Campinas-SP.

F oram sel ecionadas 42 pacientes tratadas antes da puberdade com quimioterapia sistêmica e intratecal, e com radioterapia cranial de 18 ou 24Gy. Todas já haviam apresentado telarca, das quais 40 tinham mostrado pubarca e 32, menarca. As pacientes foram subdivididas de acordo com a dose de radioterapia cranial utilizada, 18 ou 24Gy, e de acordo com a idade no início do tratamento, antes dos cinco ou com cinco ou mais anos de idade.

Como controle, utilizou-se um estudo realizado com meninas saudáveis, selecionadas do Ambulatório de Ginecologia de Adol escentes do Centro de Atenção Integral à Saúde da Mulher (CAISM). Foram obtidas informações relativas à idade de menarca em 6.608 adol escentes. Destas, 232 apresentavam informações no prontuário sobre as idades de telarca e pubarca. Os métodos estatísticos utilizados foram o teste te Student e a análise de regressão linear múltipla. As diferenças entre as variáveis foram consideradas estatisticamente significativas quando o $p$ foi menor que 0,05 .

\section{RESULTADOS}

A idade média da telarca no grupo de meninas tratadas de LLA foi de 9,8 anos, sendo estatisticamente inferior à observada no grupo-controle, que foi de 11,1 anos. As idades médias da pubarca e da menarca no grupo tratado foram inferiores às do grupo-controle, não sendo essas diferenças estatisticamente significativas (tabela 1 ).
Tabela 1 - Comparação das médias das idades na telarca, pubarca e menarca entre o grupo tratado e um grupo-controle

\begin{tabular}{|lccrc|}
\hline \multicolumn{1}{|c}{ Variável } & $\begin{array}{c}\text { Média } \\
\text { (em anos) }\end{array}$ & Desvio-padrão & $\mathbf{n}$ & $\mathbf{p}^{*}$ \\
Telarca & & & & \\
grupo tratado & 9,8 & 1,2 & 42 & $<0,001$ \\
grupo-controle & 11,1 & 1,5 & 232 & \\
$\begin{array}{l}\text { Pubarca } \\
\text { grupo tratado }\end{array}$ & 10,0 & 1,4 & 40 & $\mathrm{~ns}$ \\
grupo-controle & 11,2 & 1,5 & 232 & \\
$\begin{array}{l}\text { Menarca } \\
\text { grupo tratado }\end{array}$ & 11,6 & 1,5 & 32 & $\mathrm{~ns}$ \\
grupo-controle & 13,0 & 1,7 & 6.608 & \\
\hline
\end{tabular}

Teste $t$ de Student para amostras independentes. ns = não-significativo.

Tabela 2 - Comparação das médias das idades na telarca, pubarca e menarca, segundo a dose de radioterapia cranial (18 ou 24Gy)

\begin{tabular}{lccrc|}
\hline \multicolumn{1}{c}{ Variável } & $\begin{array}{c}\text { Média } \\
\text { (em anos) }\end{array}$ & Desvio-padrão & $\mathbf{n}$ & $\mathbf{p}^{*}$ \\
Telarca & & & & \\
RT 18 & 9,7 & 1,1 & 31 & $\mathrm{~ns}$ \\
RT 24 & 10,2 & 1,6 & 11 & \\
grupo-controle & 11,1 & 1,5 & 232 & $\mathrm{~ns}+$ \\
Pubarca & & & & \\
RT 18 & 9,9 & 1,3 & 29 & $\mathrm{~ns}$ \\
RT 24 & 10,4 & 1,6 & 11 & \\
grupo-controle & 11,2 & 1,5 & 232 & $\mathrm{~ns}+$ \\
Menarca & & & & \\
RT 18 & 11,6 & 1,3 & 21 & $\mathrm{~ns}$ \\
RT-24 & 11,6 & 1,9 & 11 & \\
grupo-controle & 13,0 & 1,7 & 6.608 & $\mathrm{~ns}+$ \\
\hline Teste $t$ de Student para amostras independentes; ns = não-significativo; \\
$+=$ grupos tratados com 18 ou 24Gy em relação ao grupo-controle. \\
\hline
\end{tabular}

Aosubdividir as meninas tratadas de LLA de acordo com a dose de radioterapia cranial utilizada (18 ou 24Gy), pôde-se observar que não houve diferenças estatisticamente significativas entre os dois grupos, quanto à telarca, pubarca e menarca (tabela 2).

Quando comparadas as meninas tratadas de acordo com a idade no início do tratamento, dividindo-as em dois grupos - início do tratamento com menos de cinco anos e com cinco ou mais anos -, observou-se que a tel arca, pubarca e menarca ocorreram mais precocemente no grupo que iniciou o tratamento antes dos cinco anos de idade. Porém, essas diferenças foram estatisticamente significativas apenas para a idade da menarca (tabela 3).

As tabelas 4, 5 e 6 evidenciaram a importância da idade por ocasião do tratamento sobre o desenvolvimento puberal: quanto mais jovem a menina, mais cedo será sua puberdade, independentemente da dose (18 ou 24Gy) de radioterapia utilizada. 
Tabela 3 - Comparação das médias das idades na telarca, pubarca e menarca, segundo idade no início do tratamento

\begin{tabular}{lcccc|}
\hline \multicolumn{1}{|c}{ Variável } & $\begin{array}{c}\text { Média } \\
\text { (em anos) }\end{array}$ & Desvio-padrão & $\mathbf{n}$ & $\mathbf{p}^{*}$ \\
$\begin{array}{l}\text { Telarca } \\
\text { Idade no início }\end{array}$ & & & & \\
do tratamento & & & & \\
$<5$ anos & 9,4 & 1,0 & 19 & $\mathrm{~ns}$ \\
$\geq 5$ anos & 10,1 & 1,3 & 23 & \\
$\begin{array}{l}\text { Pubarca } \\
\text { Idade no início }\end{array}$ & & & & \\
do tratamento & & & & \\
$<5$ anos & 9,6 & 0,8 & 18 & $\mathrm{~ns}$ \\
$\geq 5$ anos & 10,4 & 1,6 & 22 & \\
$\begin{array}{l}\text { Menarca } \\
\text { Idade no início }\end{array}$ & & & & \\
do tratamento & & & & \\
$<5$ anos & 10,9 & 1,3 & 12 & $<0,05$ \\
$\geq 5$ anos & 12,0 & 1,5 & 20 & \\
\hline Teste $t$ de Student para amostras independentes; ns = não-significativo. & \\
\hline
\end{tabular}

Tabela 4 - Fatores associados à idade na telarca: regressão linear múltipla

\begin{tabular}{|lcrc|}
\hline \multicolumn{1}{c}{ Variável } & Coeficiente & EP coeficiente & $\mathbf{p}$ \\
Idade no início & 0,2894 & 0,0669 & $<0,0002$ \\
do tratamento & 8,3351 & 0,3764 & $<0,0001$ \\
Constante & & \\
\hline
\end{tabular}

Tabela 5 - Fatores associados à idade na pubarca: regressão linear múltipla

\begin{tabular}{|lccc|}
\hline \multicolumn{1}{c}{ Variável } & Coeficiente & EP coeficiente & $\mathbf{p}$ \\
Idade no início & 0,3092 & 0,0789 & $<0,0005$ \\
do tratamento & 8,4481 & 0,4430 & $<0,0001$ \\
Constante & & & \\
\hline
\end{tabular}

Tabela 6 - Fatores associados à idade na menarca: regressão linear múltipla

\begin{tabular}{|lccc|}
\hline \multicolumn{1}{c}{ Variável } & Coeficiente & EP coeficiente & $\mathbf{p}$ \\
Idade no início & 0,3444 & 0,0838 & $<0,0005$ \\
do tratamento & 9,5918 & 0,5293 & $<0,0001$ \\
Constante & & \\
\hline
\end{tabular}

\section{DISCUSSÃO}

Nossos resultados mostraram que o desenvolvimento puberal em meninas tratadas de LLA na infância foi mais precoce que o de meninas saudáveis com as quais foram comparadas. Embora com significância estatística apenas para a telarca, as médias das idades de ocorrência da pubarca e menarca foram inferiores no grupo tratado.
O desenvolvimento puberal não foi diferente segundo a dose de radioterapia cranial utilizada. I sto pôde ser comprovado pelas médias das idades de telarca, pubarca e menarca nos dois grupos (18 e 24Gy).

Nossos resultados evidenciaram a importância da idade no início do tratamento para LLA no desenvolvimento puberal. Quando realizado antes dos cinco anos de idade, o desenvolvimento foi mais precoce. Resultados semelhantes foram encontrados em estudo inglês que observou um início mais precoce da puberdade em meninas tratadas de LLA antes dos sete anos de idade ${ }^{15}$. Em outro estudo, entretanto, os autores notaram que a idade de início da puberdade foi igual à da população geral quando o tratamento ocorreu antes dos sete anos de idade, e que houve atraso puberal quando o tratamento ocorreu após os sete anos ${ }^{7}$. O mesmo não observamos quando estudamos as meninas tratadas após os cinco anos de idade, que se comportaram de maneira semel hante ao grupo-controle. $\mathrm{O}$ menor número de meninas observadas no referido estudo de Hokken-Koel ega et al. ${ }^{7}$ pode ter sido o determinante da diferença de seus resultados em relação aos nossos.

A explicação para as alterações no desenvolvimento puberal observadas em meninas tratadas de LLA deve enfocar tanto o tratamento radioterápico quanto o quimioterápi co e a idade em que são realizados. Doses baixas de radioterapia cranial estão associadas com precoci dade sexual, provavelmente por estimulação do eixo hipotálamohipófise ${ }^{3}$. Sabe-se que a secreção do hormônio liberador de gonadotrofinas (GnRH) é controlada negativamente por áreas produtoras de opiáceos no hipotálamo e que esse controle inibitório deve ser o responsável pel o bloqueio do desenvolvimento gonadal durante a infância ${ }^{16}$. Danos nessa área inibitória, provocados pela irradiação, poderiam permitir a ativação prematura do gerador de pulsos do GnRH, determinando um aumento precoce na secreção do hormôni o fol ículo-estimulante (FSH) e do hormônio luteinizante (LH) .

Essa ação da radioterapia ocorre simultaneamente às alterações gonadais provocadas pela quimioterapia. As I esões provocadas pela quimioterapia consistem em inibição do crescimento folicular, diminuição do número de folículos primários, lesões vasculares e fibrose cortical ${ }^{17,18}$. As células gonadais de meninas pré-púberes devem ser mais suscetíveis que as das meninas mais jovens. A insuficiência gonadal pouco antes da puber dade pode-se exprimir por atraso ou lenti dão no desenvolvimento puberal, com retardo no aparecimento dos caracteres sexuais secundários ${ }^{4}$. 
Essa insuficiência pode impedir a manifestação da precocidade sexual determinada pela radioterapia. Essa deve ser a razão de termos observado que o desenvolvimento puberal foi mais tardio nas meninas tratadas após os cinco anos de idade (em relação às tratadas antes dessa idade).

As diferenças observadas no desenvolvimento puberal em nosso estudo parecem ser determinadas, principalmente, pela época em que o tratamento da LLA foi realizado. A ação da quimioterapia pode neutralizar ou contrabalançar os efeitos indutores da precocidade sexual da radioterapia, dependendo da idade em que o tratamento é realizado. Provavelmente, quando realizado após os cinco anos de idade, a quimioterapia determina lesões gonadais que não permitem resposta ovariana ao aumento de gonadotrofinas induzido pela radioterapia.

As dúvidas e controvérsias observadas após o tratamento de crianças com câncer, provavelmente, serão elucidadas quando o aumento no número de sobreviventes possibilitar maiores estudos.

\section{CONCLUSÕES}

O desenvolvimento puberal em meninas tratadas de LLA na infância foi mais precoce que o de meninas saudáveis.

A dose de radioterapia cranial utilizada não influenciou a época do desenvolvimento puberal.

As meninas tratadas antes dos cinco anos apresentaram desenvolvimento puberal mais precoce do que aquelas tratadas com cinco ou mais anos de idade.

\section{SUMMARY}

\section{Development of puberty in girls treated by acute Iymphocytic leukaemia}

BACKGROUND. In order to evaluate the puberal development of girls treated by Acute Lymphocytic Leukaemia (ALL) a retrospective study was done at Campinas-SP, Brazil.

Material and Methods. Forty two girls were treated by ALL with either 18 or 24 Grays of cranial irradiation. All patients were treated with che motherapy including intrathecal methotrexate in similar dose regimens in either groups. REsULts. The results showed lower mean ages at telarche, pubarche and menarche in the treated group, mainly in the group treated before five years old. No differences were observed in the 18 Grays or 24 Grays group.
Conclusions. Our data suggest that girls treated by ALL havea precocious puberal devel opment. [Rev Ass Med Brasil 1998; 44(3): 214-7.]

KEY WORDS: Precocius puberty. Acute lymphocytic leukaemia - treatment.

\section{REFERÊNCIAS BIBLIOGRÁFICAS}

1. Birch J M, Marsden HB, Morris-J ones PH, Pearson D, Blair V. Improvements in survival from childhood cancer in results of a population based study over 30 years. Br Med J 1988; 296: 1.372-5

2. Stiller CA, Bunch KJ . Trends in survival of childhood cancer in Britain diagnosed 1971-85. Br J Cancer 1990; 62: 806.

3. Leiper AD, Stanhope R, Preece MA, Grant DB, Chessels J M. Precocious or early puberty and growth failurein girls treated for acute lymphoblastic leukaemia. Horm Res 1988; 30: 72-6.

4. Brauner R, Thibaud E, Rappaport R. Fonction gonadique apres traitement des cancers de l'enfant. Ann Pédiatr 1990; 37: 437-43.

5. Styne DM, Melvin MG. Puberdade no homem e na mulher sua fisiologia e distúrbios. In Yen SSC, J affe RB. Endocrinol ogia reprodutiva: fisi ol ogia, fi si opatol ogia etratamento clínico. São Paulo, ed. Roca, 1990; 345.

6. Moell C, Garwicz S, Westgren U, WiebT, Albertsson-Wikland K. Blunted pubertal growth after leukemia: a new pattern of growth hormone insuficiency. Horm Res 1988; 30: 68-71.

7. Hokken-Koel ega ACS, Van Doorn J WD, Hahlen L et al. Longterm effects of treatment for acute lymphoblastic leukemia with and without cranial irradiation on growth and puberty: a comparative study. Pediatr Res 1933; 33: 577-82.

8. Quigley C, Cowell C, J imenez M et al. Normal or early development of puberty despite gonadal damage in children treated for acute lymphoblastic leukemia. New Engl J Med 1989; 321: 143-51.

9. Siris ES, Leventhal BG, Vaitukaitis J L. Effects of childhood leukemia and chemotherapy on puberty and reproductive function in girls. New Engl J Med 1976; 294: 1.143-6.

10. Blatt J , Poplack DG, Sherins RJ . Testicular function in boys after chemotherapy for acute lymploblastic leukemia. N ew Engl J Med 1981; 304: 1.121-4.

11. Chessels J M. Childhood acute lymphoblastic leukaemia: the late effects of treatment. Br J Haematol 1983; 53: 369-78.

12. Shalet SM, Beardwell CG, Twomey JA, Morris-J ones PH, Pearson D. Endocrine function following the treatment of acute leukemia in childhood. J Pediatr 1977; 90: 920-3.

13. Shalet SM. Effects of cancer chemotherapy on gonadal function of patients. Cancer Treat Rev 1980; 7: 141-52.

14. Shal et SM, Hann J M, Lendon M, Morris-J ones PH, Beardwell CG. Testicular function after combination chemotherapy in childhood for acute lymphoblastic leukaemia. Arch Dis Child 1981; 56: 275-8.

15. Uruena M, Stanhope R, Chessells J M, Leiper AD. Impaired pubertal growth in acute lymphoblastic leukaemia. Arch Dis Child 1991; 66: 1.403-7.

16. Marshall J C \& Kelch RP. Gonadotropin-releasing hormone: role of pulsatile secretion in the regulation of reproduction. New Engl J Med 1986; 315: 1.459-68.

17. Lushbaugh CC \& Casarett GW. The effects of gonadal irradiation in clinical radiation therapy: a review. Cancer 1976; 37: 1.111-20.

18. Miller JJ , Williams GF, Leissring J C. Multiple late complications of therapy with cyclophosphamide, including ovarian destruction. AmJ Med 1971; 50: 530-5. 